BRIEF COMMUNICATIONS

Evolution, 56(12), 2002, pp. 2541-2547

\title{
DIRECT AND CORRELATED RESPONSES TO ARTIFICIAL SELECTION ON DEVELOPMENTAL TIME AND WING LENGTH IN DROSOPHILA BUZZATII
}

\author{
Marcelo D. Cortese, ${ }^{1}$ Fabian M. Norry, Romina Piccinali, and Esteban Hasson ${ }^{2}$ \\ Departamento de Ecología Genética y Evolución, Facultad de Ciencias Exactas y Naturales, Universidad de Buenos Aires, \\ Ciudad Universitaria Pab. II., C1428EHA, Buenos Aires, Argentina \\ ${ }^{1}$ E-mail: mcortese@fibertel.com.ar \\ ${ }^{2}$ E-mail: ehasson@bg.fcen.uba.ar
}

\begin{abstract}
Developmental time and body size are two positively correlated traits closely related to fitness in many organisms including Drosophila. Previous work suggested that these two traits are involved in a trade-off that may result from a negative genetic correlation between their effects on pre-adult and adult fitness. Here, we examine the evolution of developmental time and body size (indexed by wing length) under artificial selection applied to one or both traits in replicated $D$. buzzatii populations. Directional changes in both developmental time and wing length indicate the presence of substantial additive genetic variance for both traits. The strongest response to selection for fast development was found in lines selected simultaneously to reduce both developmental time and wing length, probably as an expected consequence of a synergistic effect of indirect selection. When selection was applied in the direction opposite to the putative genetic correlation, that is, large wing length but fast development, no responses were observed for developmental time. Lines selected to reduce both wing length and developmental time diverged slightly faster from the control than lines selected to increase wing length and reduce developmental time. However, wing length did not diverge from the control in lines selected only for fast development. These results suggest a complex genetic basis of the correlation between developmental time and wing length, but are generally consistent with the hypothesis that both traits are related in a trade-off. However, we found that this trade-off may disappear under uncrowded conditions, with fast-developing lines exhibiting a higher pre-adult viability than other lines when tested at high larval density.
\end{abstract}

Key words. - Antagonistic pleiotropy, body size, developmental rate, genotype-by-environment interaction, pre-adult viability, trade-off.

Received April 21, 2002. Accepted August 19, 2002.

From the perspective of population genetics, constraints to the evolution of life-history traits could result from either a lack of genetic variation (i.e., no or very low heritability) or trade-offs between pleiotropically related traits. The hypothesis of trade-offs between traits often emphasizes the role of negative genetic correlations between early and late fitness components (Williams 1957; Rose 1982, 1984; Stearns 1992; Curtsinger et al. 1994; Roff 1996, 1997, 2000).

Larval developmental time and wing size (an index of adult body size) are two very important traits in Drosophila life history (Lewontin 1965; Istock 1984). A faster development is expected to increase fitness in two ways, either through a positive effect on survival under conditions of larval crowding or through its putative demographic advantage for early reproduction in expanding populations. Larger wing size, on the other hand, would be favored during the adult stage as suggested by its positive correlation with several adult fitness components (for references, see Roff 2000). Although overall body size may not always be the true target of selection (Norry et al. 1995), wing size is almost certainly under selection in the wild (Long and Singh 1995; Karan et al. 1998; Huey et al. 2000). Thus, a trade-off between either wing size or body size and developmental time is expected because the demographic and/or larval viability advantage of fast-developing individuals would be counterbalanced by their small adult size and vice versa (e.g., Partridge and Fowler 1993; Zwaan et al. 1995; Betrán et al. 1998; Fernández Iriarte and Hasson 2000).

The major aim of the present study is to examine the re- sponses to artificial selection on developmental time and wing length in the cactophilic species $D$. buzzatii. Evidence for a genetic control of the trade-off in this species comes from observed antagonistic effects of polymorphic inversions of the second chromosome on both adult size and developmental time (Betrán et al. 1998; Fernandez Iriarte and Hasson 2000). However, a basic limitation of these studies is that they only provide a static estimate of genetic components of the (co)-variances between traits in both laboratory and wild populations. In addition, short-term correlated responses to selection might, sometimes, depend not only on the genetic and phenotypic variances and covariances between the traits but also on population size, selection intensity, number of loci, allele frequencies, linkage disequilibrium, and, potentially, the details of pleiotropy (Gromko 1995; Falconer and Mackay 1996; Harshman and Hoffmann 2000). For example, asymmetrical correlated responses to selection may be a possible evolutionary outcome for physiologically complex traits (Shiotsugu et al. 1997). Additionally, selection experiments often provide a more powerful approach due to the accumulation of direct and indirect responses over generations and the possibility of obtaining estimates of among-population variation by using multiple replications within selection treatments (Rose et al. 1996).

In the present paper we address two main issues. First, we test the null hypothesis of genetic constraints due to absence of genetic variation for wing size and developmental time (i.e., no selection response), against the alternative hypotheses of a genetic correlation between traits. This is investi- 
gated by examining the pattern of direct and indirect responses to selection applied in two ways: (1) in the direction opposite to the putative genetic correlation, that is, selecting for large wing length and fast development; and (2) in the same direction of the putative genetic correlation, that is, selecting for small wing length and fast development. A second aim is motivated by the fact that correlations between traits can change across environments with different nutritional qualities (e.g., Kause and Morin 2001). Such genotypeby-environment interaction is an expected outcome from models of phenotypic plasticity (e.g., de Jong 1990; Stearns et al. 1991). Thus, given that developmental time and larval survival are expected to be strongly correlated in Drosophila as well as in other insects (see above), we also examine preadult survival differences between selection lines under different conditions of larval density.

\section{Materials AND Methods \\ Collection of Stocks}

Flies originated from 60 isofemale lines collected in Otamendi, Buenos Aires Province, Argentina (for a description of the site of collection; see Hasson et al. 1995), where $D$. buzzatii breeds and feeds on the rotting cladodes of the prickly pear Opuntia vulgaris. Five males and five females of the offspring of each isofemale line were collected as virgin adults and mixed in two cages, one for each sex. From these cages, 20 groups of 15 flies of each sex were made to set up five sets of four lines each. One set was used to obtain mean values of wing length in the base population (B) and the remaining four sets were assigned arbitrarily to each one of the three selection treatments and the control (see below).

During the course of the experiment, all replicated lines were kept synchronized and continuously maintained under uncrowded conditions by allowing the parents of each generation to mate and oviposit for no longer than $72 \mathrm{~h}$. Cultures were raised in a temperature controlled room $\left(25 \pm 1^{\circ} \mathrm{C}\right)$ in $100-\mathrm{ml}$ bottles with $30 \mathrm{ml}$ of a modified formula of killedyeast Drosophila medium (David 1962), hereafter called standard bottles.

\section{Selection Lines and Controls}

Directional selection on wing length and/or developmental time was applied for 22 generations in four replicated lines in each of the following four treatments:

(1) Fast development and large wing length (L lines): in each replicate, the first 25 pairs (of approximately 150 flies) that emerged from each culture bottle were scored for wing length as described below, and the 10 males and 10 females with the highest scores were selected as parents of the next generation.

(2) Fast development and small wing length (S lines): same procedure as in (1), with the only difference that the individuals selected as parents were those with the lowest wing-length scores.

(3) Fast development (F lines): ten of the first 25 emerged flies of each sex were randomly chosen as parents of the next generation. The purpose of this treatment is twofold. First, it can be used for the assessment of the direct response to selection on developmental time (see below). Second, it is a control for combined developmental time and wing-length selection regimes ( $\mathrm{L}$ and $\mathrm{S}$ lines).

(4) Overall control, with no selection treatment (C lines): ten flies of each sex were randomly chosen as parents of the next generation, from the total of emerged flies. These lines can be considered as a general control because no selection treatment was applied.

\section{Generations Sampled for the Analysis of Direct and Correlated Responses}

Twenty virgin flies of each sex were randomly sampled from the offspring of each replicate in the base population as well as in generations 10 and 22. Sampled flies were released into mating cages (one per replicate) and allowed to mate for $12 \mathrm{~h}$ and oviposit for $24 \mathrm{~h}$ in petri dishes containing an oviposition medium (agar, acetic acid, and yeast). Dishes were removed and incubated for $24 \mathrm{~h}$ at $25^{\circ} \mathrm{C}$ to allow egg hatching. Batches of 100 one- to two-hour-old larvae were transferred to standard bottles and incubated at $25 \pm 1{ }^{\circ} \mathrm{C}$ until adult emergence.

In addition, five virgin flies of each sex and replicate within treatments were sampled and released into mating cages (one cage per treatment). This procedure was employed to obtain $F_{1}$ hybrids among lines within treatments (hereafter called $F_{1}$ hybrids) to test for the effect of inbreeding due to the limited number of parents used in each generation.

The number and sex of all flies emerged in each culture bottle were scored every $3 \mathrm{~h}$ for generations 10 and 22. Developmental time was taken as the midpoint between successive scorings. Wing length was measured as the distance between the intersection of the second and third veins and the distal end of the latter. One randomly chosen wing was scored. Measurements were performed with a binocular microscope fitted with an ocular micrometer (1 ocular unit $=$ $0.025 \mathrm{~mm})$ at $40 \times$ magnification.

We also investigated the correlated response to selection of larval survival in S, L, and C lines in generation 11. Hybrid larvae were obtained from crosses among replicates as described above for developmental time. First instar larvae were transferred to vials containing $10 \mathrm{ml}$ of a seminatural culture medium prepared with liquefied fresh cladodes of Opuntia vulgaris. Five replicates with 40 larvae per vial (low density) and five replicates with 200 larvae per vial (high density) were set up for each selection treatment. Vials were kept at $25 \pm 1{ }^{\circ} \mathrm{C}$ until the emergence of all flies. Larval survival was estimated as the number of flies emerging in each vial relative to the number of larvae seeded.

\section{Statistical Analysis}

Both developmental time and wing length were analyzed by means of three-way ANOVAs, with selection treatment and sex as fixed factors and replicate as a random factor within treatments. Differences between treatments were tested by means of pairwise comparisons between means using Tukey's test (Sokal and Rohlf 1995).

Means of $F_{1}$ hybrids were also compared with three-way ANOVAs, with sex, selection treatment, and the condition hybrid versus non-hybrid as fixed factors. Tukey pairwise 


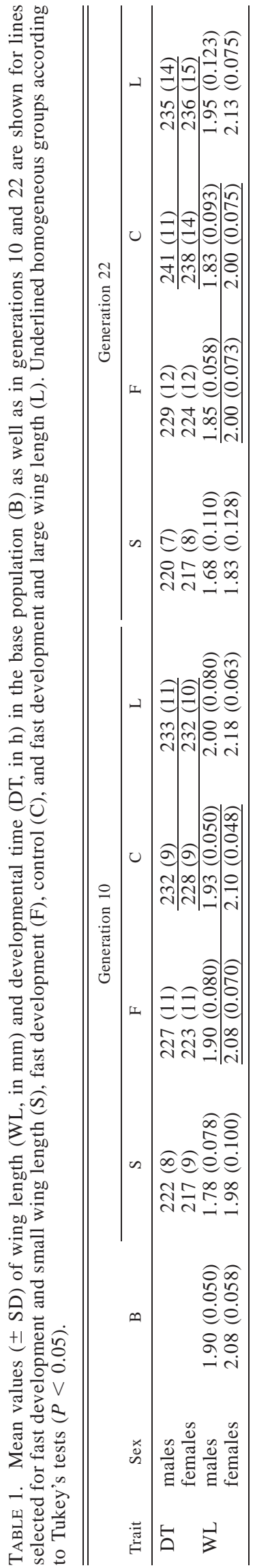

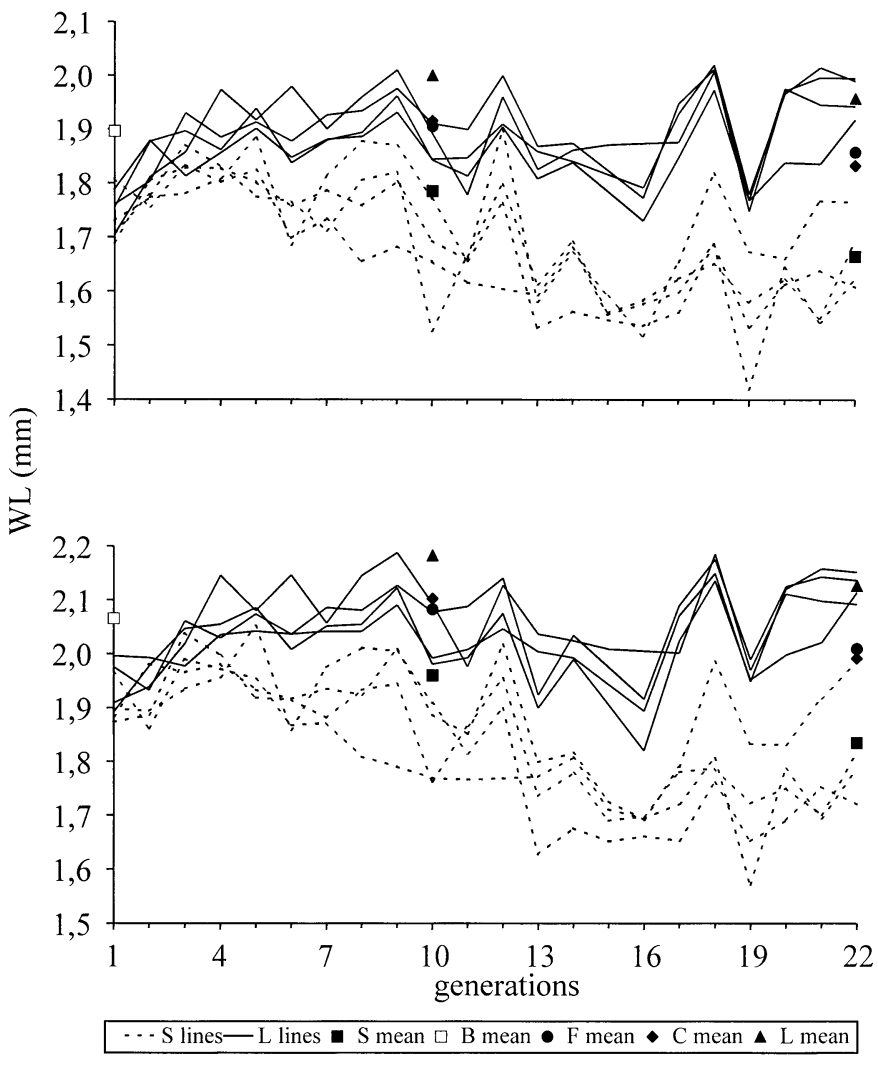

FIG. 1. Experimental evolution of wing length in $\mathrm{S}$ and $\mathrm{L}$ lines under divergent selection for males (upper panel) and females (lower panel). Mean values for individuals reared under standardized conditions of larval density are shown for the base population as well as for S, L, F, and control lines in generations 10 and 22 of selection.

comparisons were also performed when significant factors or interactions were found.

Viability data (expressed as proportions) were transformed using the arcsine-square root transformation (Sokal and Rohlf 1995), and analyzed with a two-way ANOVA, using density and selection treatment as fixed factors. Statistical significance of the differences among group means was tested with Tukey's test.

Finally, evolution of wing length was analyzed by means of regression analysis on time (generations) for both $\mathrm{L}$ and $\mathrm{S}$ treatments. The absolute values of the regression coefficient were compared using a $F$-test of the differences between regression coefficients (Sokal and Rohlf 1995).

The program package Statistica 1999 (StatSoft Inc., Tulsa, OK) was used for all statistical analyses.

\section{RESULTS}

Mean values of both developmental time and wing length are given in Table 1. Experimental evolution of wing length is shown for $\mathrm{S}$ and $\mathrm{L}$ lines in Figure 1. An inspection of mean values in generations 10 and 22 shows that the lines selected for increased versus decreased wing length evolved in opposite directions (Fig.1), even though both lines were simultaneously selected for fast development. Differences among lines are highly significant in both generations 10 
TABLE 2. Analyses of variance performed to test for differences in developmental time and wing length among selection lines and control for 22 generations of selection.

\begin{tabular}{|c|c|c|c|}
\hline Effect & $\mathrm{df}$ & MS & $F$ \\
\hline \multicolumn{4}{|l|}{ DT } \\
\hline Treatment (line) & 3 & 22894.67970 & $13.2 * *$ \\
\hline Replicate within treatment & 12 & 1736.02417 & $14.4 * *$ \\
\hline Treatment $\times$ sex & 3 & 209.67345 & 0.9 \\
\hline Replicate within treatment $\times$ sex & 12 & 226.68549 & $1.9 *$ \\
\hline Within & 1002 & 120.23902 & \\
\hline Replicate within treatment & 12 & 0.09269 & $13.9 * *$ \\
\hline Sex & 1 & 3.03475 & $322.0 * *$ \\
\hline Treatment $\times$ sex & 3 & 0.00206 & 0.2 \\
\hline Replicate within treatment $\times$ sex & 12 & 0.00944 & 1.4 \\
\hline Within & 474 & 0.00669 & \\
\hline
\end{tabular}

$* P<0.05 ; * * P<0.001$.

$\left(F_{3,12}=87.625, P<0.001\right)$ and 22 (Table 2$)$, despite significant variation among replicates within selection treatments. Multiple comparisons using Tukey's test confirm that $\mathrm{S}$ and $\mathrm{L}$ lines differ significantly $(P=0.00016)$, and that these two lines differ from $\mathrm{F}$ and $\mathrm{C}$ (Table 1).

An asymmetrical response to selection is apparent for wing length, as S lines appear to diverge faster than L lines in both sexes when reared under our selection conditions (Fig. 1). This trend was found after comparing the slope of the regressions of wing length on generations. Regressions are highly significant for both selection treatments $(b=-0.62$, $P<0.001$ for $\mathrm{S}$ lines and $b=0.25, P<0.001$ for $\mathrm{L}$ lines), and the absolute values of the regression coefficients are significantly different $(F$-test for the difference between regression coefficients: $\left.F_{1,336}=22.48, P<0.001\right)$. However, this asymmetry is less evident relative to the controls $(C)$ in the samples of generations 10 and 22 reared under low and more controlled larval density (Table 1; Fig. 1). F lines, selected only for fast development, do not differ in wing length from the control C (Table 1; Fig. 1).

Developmental time also shows a substantial response when selected for alone (F lines) or in conjunction with selection for small wing length (S lines), but not when wing length is selected to increase its size (L lines, Table 1). This indicates a considerable amount of additive genetic variance for developmental time, despite of a lack of response in $\mathrm{L}$ lines. Differences in developmental time among selection treatments are significant (Table 2 for generation $22 ; F_{3,12}=$ 6.785, $P<0.001$ for generation 10 ), and mainly due to the divergence of $\mathrm{F}$ and $\mathrm{S}$ from $\mathrm{C}$ and $\mathrm{L}$ (Table 1).

In average, developmental time increased in the lab, with controls ( $\mathrm{C}$ lines) developing faster in generation 10 than in generation 22 (Table 1). This apparent change in the control lines demands a critical survey of the data. This indicates that all selection lines were also subject to some sort of adaptation to the laboratory environment, which is known to increase developmental time (Sgrò and Partridge 2000). Therefore, the apparent stability in $\mathrm{S}$ and $\mathrm{F}$ lines between generations 10 and 22 should not be interpreted as a lack of response to selection for fast development in this time interval. However, this experimental result cannot place any doubt on the observation that the $\mathrm{S}$ and $\mathrm{F}$ lines diverged in developmental time (Table 1). Indeed, selection responses in developmental time are clearly dependent on the selection regime on wing length. On one hand, developmental time diverged significantly from the control in both $\mathrm{S}$ lines (selected for small wing length) and $F$ lines (not selected for wing length), and the magnitude of this response is larger for $\mathrm{S}$ lines. On the other hand, L lines (selected for large wing length) did not diverge from the control, $\mathrm{C}$ (Table 1).

$\mathrm{F}_{1}$ hybrids versus non-hybrids were compared to test for inbreeding effects. Inbreeding depression is apparent in developmental time (Fig. 2 A, B), as F1 hybrids tend to develop significantly faster than nonhybrids in generations $10\left(F_{1,1369}\right.$ $=21.7, P<0.001)$ and $22\left(F_{1,1316}=23.6, P<0.001\right)$. The significant interaction between selection treatment and the hybrid versus nonhybrid condition in generation $22\left(F_{3,1316}\right.$ $=4.2, P<0.01)$ seems to be mainly due to the nonsignificant differences between hybrids and nonhybrids in $\mathrm{S}$ lines (Fig. $2 \mathrm{~B} ; P=0.78)$. In contrast to developmental time, both the hybrid versus nonhybrid factor in wing length and its interaction with selection treatment are significant in generation $22\left(F_{1,630}=24.0, P<0.001\right.$ and $F_{3,630}=2.7, P<0.05$; Fig. 2D) but not in generation 10 (Fig. 2C). The results of multiple comparisons using Tukey's test suggest that the significance is mainly due to the difference between $\mathrm{F}_{1}$ hybrids and nonhybrids among $\mathrm{L}$ lines $(P=0.00011)$.

Pre-adult survival was compared among selection treatments in cultures started with high (200 individuals per vial) versus low (40 individuals per vial) density. We found a strong interaction $\left(F_{1,24}=4.11, P<0.05\right)$ between selection treatment and larval density (Fig. 3). S lines show a higher viability than L at high density (Fig. 3; $P=0.043$ for Tukey's test), and a lower, but not significant, viability than L lines at low density (Fig. 3; Tukey comparison: $P=0.08$ ).

\section{DISCUSSION}

Both developmental time and wing length responded to selection in D. buzzatii, with $\mathrm{S}$ lines showing the strongest response and diverging from controls by almost $10 \%$ in generation 22. These results indicate that additive genetic var- 

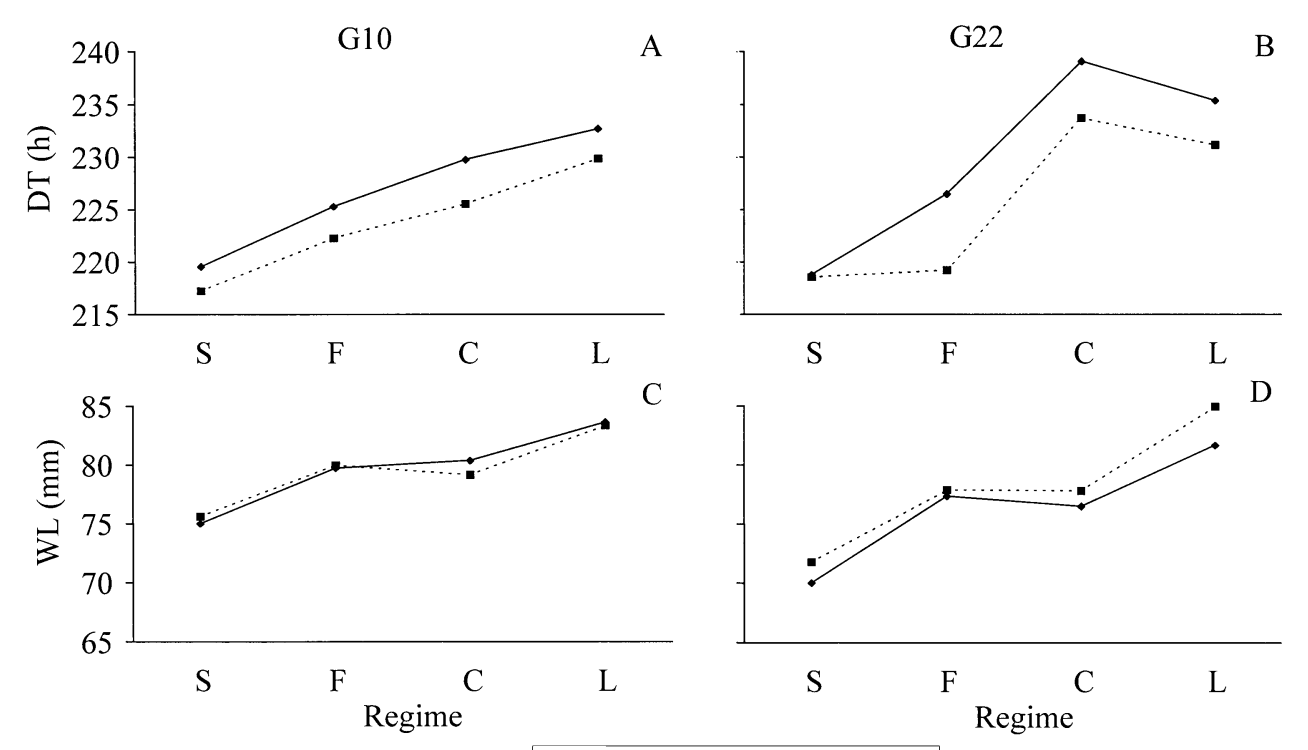

-non-hybrids ..F1 hybrids

FIG. 2. Developmental time (A, B) and wing length $(C, D)$ are shown for $F_{1}$ hybrids among replicated lines within treatments (dotted lines) and for nonhybrid lines (continuous lines) in generations $10(\mathrm{~A}, \mathrm{C})$ and 22 (B, D) of selection.

iation may be substantial even for life-history traits like developmental time, which usually exhibit lower levels of additive variance than other traits (Roff 1997). Selection experiments in $D$. melanogaster have also demonstrated a significant component of additive genetic variance for developmental time and size-related traits (Hillesheim and Stearns 1991; Partridge and Fowler 1993; Zwaan et al. 1995; Nunney 1996; Chippindale et al. 1997). Our present findings suggest that developmental time and wing length are not at an evolutionary maximum as might be expected if both traits are involved in a trade-off. Specifically, L lines have evolved in the direction of increased size, whereas $\mathrm{S}$ lines evolved toward faster development, indicating that some mechanism other than absence of genetic variation may be constraining the evolution of these traits in nature.

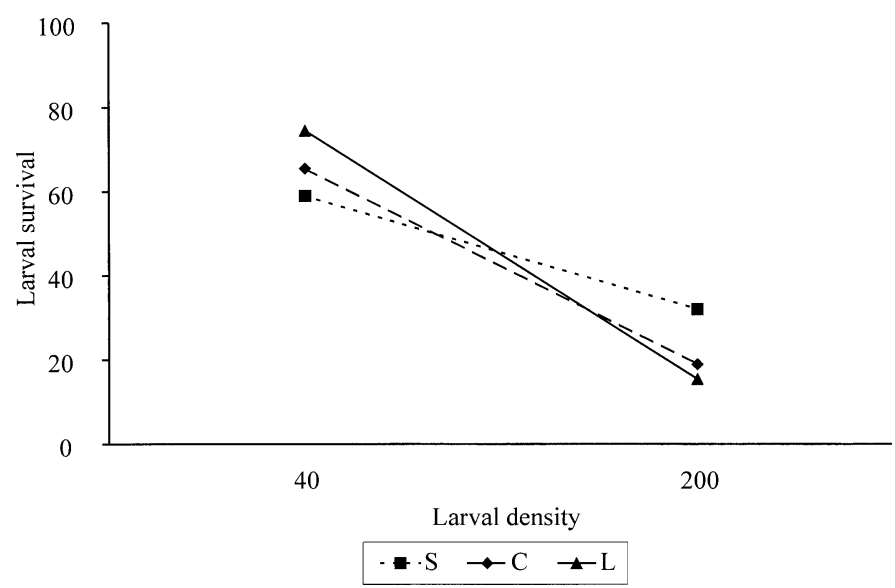

FIG. 3. Larval viability of $F_{1}$ hybrids among lines within treatments in high versus low density in generation 11 of selection.

\section{Responses to Selection of Developmental Time}

Although selection for faster development was applied in all S, L, and F lines, the response was not independent of the selection regime applied on wing length. Thus, $\mathrm{S}$ and $\mathrm{L}$ lines represent the two extremes for this trait, developmental time decreased in the former, and no change was observed in the latter. This lack of response in L lines is consistent with the hypothesis of a genetic constraint, particularly if inbreeding effects cannot per se account for these results as discussed below.

Inbreeding is usually an unavoidable by-product in artificial selection experiments due to the limited number of individuals selected as parents in each generation (Rose et al. 1996). Life-history traits (like developmental time) are typically more susceptible to inbreeding than morphological traits (like wing length), which are less closely related to fitness (DeRose and Roff 1999). Therefore, it could be argued that the apparent patterns of asymmetrical response to selection on developmental time is, in fact, a consequence of inbreeding due to a small effective population size (Falconer and Mackay 1996). Even though inbreeding effects are noticeable for developmental time, three lines of evidence indicate that inbreeding cannot per se account for the differences between $\mathrm{L}$ and $\mathrm{S}$ lines. First, the only difference between $\mathrm{L}$ and $\mathrm{S}$ lines was the direction of selection applied on wing length. The level of inbreeding in both types of lines is expected to be similar since both treatments involved the same number of parents in each generation. Second, all four replicated $\mathrm{S}$ lines responded to developmental time selection in the same direction, in sharp contrast to L lines for which no response was found. Third, the $\mathrm{F}_{1}$ hybrids among lines within treatments showed the same pattern of divergence from the control as the nonhybrids. Additionally, two observations point out that selection in $\mathrm{S}$ lines was strong 
enough to overcome the effects of genetic drift, probably due to the fixation of the same nondeleterious alleles in all four replicated S lines: (1) developmental time was not different from their respective $F_{1}$ hybrids, and (2) phenotypic variance among replicates was almost half of the value observed in all remaining experimental lines (Table 1). Therefore, the more likely explanation for the stronger response in $\mathrm{S}$ lines should be the expected synergistic effect of direct and indirect selection on developmental time and wing length, respectively, which is consistent with static estimates of the genetic correlation between developmental time and wing length in D. buzzatii (e.g., Betrán et al. 1998). Similarly, the lack of response of $\mathrm{L}$ lines is an expected outcome of the antagonistic effect of selection applied on developmental time and wing length, as noted above.

\section{Responses to Selection of Wing Length}

We observed a slight asymmetry in the evolution of wing length, as $\mathrm{L}$ and $\mathrm{S}$ lines diverged from controls in opposite directions by about $4 \%$ and $7 \%$, respectively, in generation 10. This asymmetrical response is more likely due to the indirect effect of selection applied on developmental time than to inbreeding since the latter does not substantially affect size-related traits in Drosophila (Kidwell and Kidwell 1966), and inbreeding effects were apparent only in generation 22 but not in generation 10. These results seem consistent with a positive genetic correlation between developmental time and wing length, though a possible caveat for this conclusion is that selection on wing length could be stronger in $S$ than in L lines if small flies tend to emerge first. However, under this alternative hypothesis, wing length should have evolved in the direction of small size in F lines, which was not the case in spite of what could also be predicted from any static estimates of a positive genetic correlation.

In contrast to the strong indirect selection response observed in developmental time, the rather weak or absent indirect selection response in wing length indicates that the genetic correlation between these traits is less than perfect: selection for wing length has a substantial effect on developmental time (see above), but the reverse is not true. Artificial selection studies in other insects also found this kind of asymmetry in correlated responses of traits related to early and late fitness components. For instance, lines of the bean weevil (Acanthoscelides obtectus) selected for slow developmental time live longer than controls, but lines selected for extended longevity did not increase developmental time (Tucic et al. 1998).

Our weak correlated response of body size to selection for fast development is in contrast with studies in D. melanogaster showing a correlated reduction in dry body weight (Zwaan et al. 1995, Nunney 1996, Prasad et al. 2000), and is only in partial agreement with other reports showing no reduction in thorax length in flies selected for fast development (Partridge and Fowler 1993). However, in the latter study no differences in developmental time were observed between control lines selected only for fast development and lines simultaneously selected for small thorax length and fast development. The comparison of these studies in D. melanogaster with our results in D. buzzatii suggests that some details of the genetic correlation between developmental time and body size may be species-specific, an observation that partly wains against generalizations based on studies of a single species. Perhaps the asymmetrical correlated response we found may be consistent with the recent observation that the second chromosome polymorphic inversions in $D$. buzzatii are not only involved in the trade-off but also that their average effects are much larger on developmental time than on wing length (Betrán et al. 1998; Fernández Iriarte and Hasson 2000).

\section{Genotype-by-Environment Interaction in the Trade-off}

Theory predicts that changes in genetic correlations between traits can occur between samples, species, populations, developmental stages, and even between environments within populations (Stearns et al. 1991). However, examples illustrating the influence of heterogeneous environments on a trade-off between developmental time and pre-adult survival are still scarce. In the present study the observed correlated responses to selection on developmental time suggest the importance of a genotype-by-environment interaction in the trade-off. Differences in larval survival between $\mathrm{S}$ and L lines were largely dependent on density, illustrating how tradeoffs may appear and disappear because of genotype-by-environment interactions (Stearns et al. 1991; Rose et al. 1996; Norry and Loeschcke 2002). The expected negative correlation between developmental time and larval survival was only observed at high density, whereas at lower density this correlation disappeared, or even changed in sign. Under high larval density there is increased pre-adult selection against genetically large and slow developing individuals because of their low viability, which is counterbalanced by their increased adult fitness (Santos et al. 1994; Roff 1997; Partridge and French 1996). In contrast, at low density, selection against slow pre-adult development is relaxed, and genetically larger individuals may be favored, as no such trade-off is present. Interestingly, Hillesheim and Stearns (1991) also found correlated responses of pre-adult development to selection on body weight under poor larval food conditions but not under rich conditions.

Experimental evolution in D. melanogaster showed that fly populations selected for either increased thorax length (Partridge and Fowler 1993) or slow pre-adult development (Zwaan et al. 1995) exhibit reduced pre-adult viability in high larval density. In contrast, a reduction of larval viability was reported in lines selected for fast development reared at low larval density (Chippindale et al. 1997). Such apparent differences may result from genotype-by-environment interactions between developmental time and larval density as suggested in the present paper.

\section{ACKNOWLEDGMENTS}

We thank T. Mackay, J. S. F. Baker, V. Loeschcke, A. Fontdevila, M. Santos, M. J. Hercus, T. Kawecki, and two anonymous reviewers for constructive criticism that helped to improve earlier versions of this manuscript. C. Rodríguez and J. J. Fanara and T. Kawecki are greatly acknowledged for valuable suggestions and help in different stages of this research. RP is fellow and FMN and EH are members of 
Carrera del Investigador Cientifico of Consejo Nacional de Investigaciones Cientificas y Técnicas (CONICET), Argentina. This work was supported by Universidad de Buenos Aires and CONICET grants to EH.

\section{Literature Cited}

Betrán, E., M. Santos, and A. Ruiz. 1998. Antagonistic pleiotropy effect of second-chromosome inversions on body size and early life-history traits in Drosophila buzzatii. Evolution 52:144-154.

Chippindale, A. K., J. A. Alipaz, H. W. Chen, and M. Rose. 1997. Experimental evolution of accelerated development in Drosophila. I. Developmental speed and larval survival. Evolution 51: 1536-1551.

Curtsinger J. W., P. M. Service, and T. Prout. 1994. Antagonistic pleiotropy, reversal of dominance and polymorphism. Am. Nat. 144:210-228.

David, J. 1962. A new medium for rearing Drosophila in axenic conditions. Dros. Inf. Serv. 36:128.

de Jong, G. 1990. Quantitative genetics of reaction norms. J. Evol. Biol. 3:447-468.

DeRose, M. A., and D. A. Roff. 1999. A comparison of inbreeding depression in life-history and morphological traits in animals. Evolution 53:1288-1292.

Falconer, D. S., and T. F. Mackay. 1996. Introduction to quantitative genetics. 4th ed. Longman, England.

Fernández Iriarte, P., and E. Hasson. 2000. The role of the use of different host plants in the maintenance of the inversion polymorphism in the cactophilic Drosophila buzzatii. Evolution 54: 1295-1302.

Gromko, M. H. 1995. Unpredictability of correlated responses to selection-pleiotropy and sampling interact. Evolution 49: 425-433.

Harshman, L. G., and A. A. Hoffman. 2000. Laboratory selection experiment using Drosophila: what do they really tell us? Trends Ecol. Evol. 15:32-36.

Hasson, E., C. Rodríguez, J. J. Fanara, H. Naveira, O. A. Reig, and A. Fontdevila. 1995. Macrogeographic patterns in the inversion polymorphisms of Drosophila buzzatii in New World populations. J. Evol. Biol. 8:369-384.

Hillesheim, E., and S. C. Stearns. 1991. The responses of Drosophila melanogaster to artificial selection on body weight and its phenotypic plasticity in two larval food environments. Evolution 45:1909-1923.

Huey, R. B., G. W. Gilchrist, M. L. Carlson, D. Berrigan, and L. Serra. 2000. Rapid evolution of a geographic cline in an introduced fly. Science 287:308-309.

Istock, C. A. 1984. Boundaries to life history variation and evolution. Pp. 142-168 in P. W. Price, C. N. Slobodchi-Koff, and W. S. Gaud, eds. A new ecology-novel approaches to interactive systems. John Wiley, New York.

Karan, D., A. K. Munjal, P. Gibert, B. Moreteau, R. Parkash, and J. R. David. 1998. Latitudinal clines for morphometrical traits in Drosophila kikkawai: a study of natural populations from the Indian subcontinent. Genet. Res. 71:31-38.

Kause, A., and J. P. Morin. 2001. Seasonality and genetic architecture of development time and body size of the birch feeding sawfly Priophorus pallipes. Genet Res. 78:31-40.

Kidwell, J. F., and M. M. Kidwell. 1966. The effects of inbreeding on body weight and abdominal chaeta number in Drosophila melanogaster. Can. J. Genet. Cytol. 8:207-215.

Lewontin, R. C. 1965. Selection for colonizing ability. Pp. 77-94 in H. G. Baker and G. L. Stebbins, eds. The genetics of colonizing species. Academic Press, New York.

Long, A. D., and R. S. Singh. 1995. Molecules versus morphology: the detection of selection acting on morphological characters along a cline in Drosophila melanogaster. Heredity 74:569-581.

Norry, F. M., J. C. Vilardi, J. J. Fanara, and E. Hasson. 1995.
Courtship success and multivariate analysis of sexual selection on morphometric traits in Drosophila buzzatii. J. Insect Behav. 8:219-229.

Norry, F. M., and V. Loeschcke. 2002. Temperature-induced shifts in associations of longevity with body size in Drosophila melanogaster. Evolution 56:299-306.

Nunney, L. 1996. The response to selection for fast larval development in Drosophila melanogaster and its effect on adult weight: an example of a fitness trade-off. Evolution 50: 1193-1204.

Partridge, L., and K. Fowler. 1993. Responses and correlated responses to artificial selection on thorax length in Drosophila melanogaster. Evolution 47:213-226.

Partridge, L., and V. French. 1996. Thermal evolution of ectotherm body size: why get big in the cold. Pp. 265-292 in I. A., Johnston and Albert F. Bennett, eds. Animals and temperature: phenotypic and evolutionary adaptation. Cambridge Univ. Press, Cambridge, U.K.

Prasad, N. G., M. Shakarad, V. M. Gohil, V. Sheeba, M. Rajamani, and A. Joshi. 2000. Evolution of reduced pre-adult viability and larval growth rate in laboratory populations of Drosophila melanogaster selected for shorter development time. Genet. Res. 76:249-259.

Roff, D. A. 1996. The evolution of correlations: an analysis of patterns. Evolution 50:1392-1403.

1997. Evolutionary quantitative genetics. Chapman and Hall. New York.

2000. Trade-offs between growth and reproduction: an analysis of the quantitative genetic evidence. J. Evol. Biol. 13: 434-445.

Rose, M. R. 1982. Antagonistic pleiotropy, dominance and genetic variation. Heredity 48:63-78.

- 1984. Laboratory evolution of postponed senescence in Drosophila melanogaster. Evolution 38:1004-1010.

Rose, M. R., T. J. Nusbaum, and A. K. Chippindale. 1996. Laboratory evolution: the experimental wonderland and the Cheshire cat syndrome. Pp. 221-242 in M. R. Rose and G. V. Lauder, eds. Adaptation. Academic Press, London.

Santos, M., K. Fowler, and L. Partridge. 1994. Gene-environment interaction for body size and larval density in Drosophila melanogaster: an investigation of effects on development time, thorax length and adult sex ratio. Heredity 72:515-521.

Sgró, C. M., and L. Partridge. 2000. Evolutionary responses of life history of wild-caught Drosophila melanogaster to two standard methods of laboratory culture. Am. Nat. 156:341-353.

Shiotsugu J., A. M. Leroi, H. Yashiro, M. R. Rose, and L. D. Mueller. 1997. The symmetry of correlated selection responses in adaptive: an experimental study using Drosophila. Evolution 51:163-172.

Sokal, R. R., and F. J. Rohlf. 1995. Biometry. 3rd ed. Freeman, New York.

Stearns, S. C. 1992. The evolution of life histories. Oxford Univ. Press, New York.

Stearns, S. C., G. de Jong, and B. Newman. 1991. The effects of phenotypic plasticity on genetic correlations. Trends Ecol. Evol. 6:122-126.

Tucic, N., I. Gliksman, D. Seslija, O. Stijkovik, and D. Milanovic. 1998. Laboratory evolution of life-history traits in the bean weevil (Acanthoscelides obtectus): the effect of selection on developmental time in populations with different previous history. Evolution 52:1713-1725.

Williams, G. C. 1957. Pleiotropy, natural selection, and the evolution of senescence. Evolution 11:398-411.

Zwaan, B., R. Bijlsma, and R. F. Hoekstra. 1995. Artificial selection for developmental time in Drosophila melanogaster in relation to the evolution of aging: direct and correlated responses. Evolution 49:635-648.

Corresponding Editor: T. Kawecki 